\title{
Spreads de la deuda privada y riesgo sistemático en México
}

\section{César Gurrola Ríos}

Profesor, Facultad de Economía, Contaduría y Administración, Universidad Juárez del Estado de Durango cgurrola@ujed.mx

\section{Francisco López}

\section{Herrera}

Investigador, División de Investigación, Facultad de Contaduría y Administración, UNAM francisco_lopez_herrera@yahoo.com.mx

\section{Resumen}

Este artículo ofrece evidencia empírica del comportamiento del spread o prima de riesgo de la deuda de empresas mexicanas como consecuencia del riesgo sistemático en México, capturado por la dinámica de variables macroeconómicas locales. Se propone una metodología alternativa para calcular esas primas, la cual depende únicamente de información disponible para toda empresa. Los resultados de la estimación econométrica del modelo dinámico especificado, muestran que todos los factores de riesgo propuestos, ya sea en sus valores contemporáneos o rezagados, son importantes para explicar el comportamiento de las primas de riesgo de la deuda de las emisoras bajo estudio. Dichas primas están determinadas principalmente por la evolución de las importaciones y del tipo de cambio que impactan al $100 \%$ y $96.43 \%$ de las emisoras analizadas, respectivamente. Los factores de riesgo de menor importancia son el comportamiento de las exportaciones y de los precios del petróleo que impactan, respectivamente, al $89.29 \%$ y $78.57 \%$ de la muestra.

Palabras clave: spread de crédito, prima por riesgo, riesgo sistemático. 


\title{
Private debt spreads and systematic risk in Mexico
}

\begin{abstract}
This paper offers empirical evidence about the behavior of the risk premium (spread) on debt of Mexican firms as a result of systematic risk in Mexico, captured by the dynamics of local macroeconomic variables. We posit an alternative methodology to measure such risk premia, which depends only on data available for any firm. The result of the dynamic econometric model specified, show that all the risk factors proposed, either in current or lagged values, are relevant to explain the behavior of risk premia on debt for firms in this study. These premia are determined primarily by the evolution of imports and the exchange rate, which respectively impact the $100 \%$ and $96.43 \%$ of the analyzed firms. The less significant risk factors are exports and oil prices dynamics which impact $89.29 \%$ and $78.57 \%$ of the sample, respectively.
\end{abstract}

Keywords: credit spread, risk premium, systematic risk.

\section{Introducción}

En la actualidad la medición y administración del riesgo crediticio es una tarea por demás importante para los administradores financieros, como lo muestra la creciente literatura académica que se ha generado al respecto. La evidencia disponible revela que los grados de exposición al riesgo de incumplimiento han aumentado llegando a reflejarse no sólo en el aumento de las primas de riesgo, sino en descalabros financieros de impacto internacional.

En la literatura se muestran esfuerzos ${ }^{1}$ por desarrollar modelos que permitan capturar la capacidad de pago y relacionarla con probabilidades de que cambie en el tiempo (Gentry, Shaw, Tessmer y Whitford, 2002; Benell, Crabbe, Thomas y Gwilym, 2006; Wendin y McNeil, 2006). Tales estudios utilizan análisis estadísticos univariados, discriminante múltiple, lineal de probabilidades, logit y probit, procesos de ajuste parcial, árboles de decisión, teoría de la ruina del jugador, redes neuronales, algoritmos genéticos, flujos de efectivo. Si bien es cierto que tales modelos pueden ofrecer respuestas interesantes, es preciso señalar que presentan desventajas y limitaciones. Entre las principales, no existe un acuerdo general en

\footnotetext{
${ }^{1}$ Desde los trabajos pioneros de Winakor en 1931, Hickman en 1958, Beaver en 1966, Altman en 1968 y Ohlson en 1980.
} 
cuanto a lo siguiente: a) la metodología empleada en la modelación (Gilbert, Menon y Schartz, 1990; Crouhy, Galai y Mark, 2000; Löffler, 2004); b) las variables utilizadas (Gentry, Shaw, Tessmer y Whitford, 2002; Gilbert, Menon y Schartz, 1990); c) el tipo de análisis estadístico empleado (Crouhy, Galai y Mark, 2000; Chan y Jegadeesh, 2004).

Uno de los indicadores más utilizados sobre el riesgo de crédito es la calificación crediticia o rating, calculada por agencias calificadoras independientes ${ }^{2}$. Algunos autores afirman que la complejidad de los mercados financieros ha provocando que inversionistas y reguladores incrementen su confianza en la opinión de tales agencias (Crouhy, Galai y Mark, 2001; Krahnen y Weber, 2001; Sy, 2003, Benell, Crabbe, Thomas, y Gwilym, 2006; Löffler, 2004; Hull, Predescu y White, 2004). No obstante, también hay críticas importantes hacia el trabajo de las agencias calificadoras: lentitud para incorporar el dinamismo de la economía, conflictos de agencia, falta de competencia, asimetría de información, entre otras (Livingston, Naranjo y Zhou, 2005; Chan y Jegadeesh, 2004; Crouhy, Galai y Mark, 2001; Prysock, 2006; Cantor, 2004; Krahnen y Weber, 2001; Partnoy, 2001). Adicionalmente, es importante señalar que el proceso de calificación o rating seguido por las agencias especializadas resulta un proceso restrictivo para muchas empresas; ya sea por los recursos monetarios necesarios para obtenerla o, bien, por el tipo y cantidad de información requerida en el análisis de la calidad crediticia de la empresa que desea ser calificada.

Entre las alternativas que han intentado resolver algunas de estas limitaciones destacan, por un lado, la propuesta de considerar los spreads de crédito ${ }^{3}$ en lugar de los ratings, como una aproximación de la capacidad de endeudamiento (Cantor, 2004; Crouhy, Galai and Mark, 2000; Partnoy, 2001). Lo anterior bajo el argumento que en la medida en que las probabilidades de incumplimiento aumentan, el spread tiende a incrementarse, compensando a los acreedores por el riesgo adicional. Por otro lado, se señala la necesidad de considerar el impacto del riesgo sistemático sobre la calidad crediticia y, por consecuencia, en el spread de crédito (Segoviano y Padilla, 2006; Hanson and Schuermann, 2006; Wendin y McNeil, 2006; Sy, 2003; Crouhy, Galai y Mark, 2001; De la Calle, 1991; López, 2006a y 2006b; Navarro y Santillán, 2001; López y Vázquez, 2002).

\footnotetext{
${ }^{2}$ Standard \& Poor's, Moody`s Investors Service y Fitch, entre las principales.

${ }^{3}$ Diferencia entre el rendimiento de un bono corporativo, riesgoso, y un instrumento libre de riesgo.

${ }^{4} \mathrm{Que}$ no es otra cosa que una prima por riesgo.
} 
La importancia del riesgo del crédito se puede analizar desde dos perspectivas; por un lado, en función de la eficiente asignación de recursos a los mejores usos; ; por otro, ante la posibilidad de detectar alertas tempranas o señales de deterioro que permitan acciones correctivas para evitar el riesgo de incumplimiento y, en última instancia, la quiebra. Para los practicantes del campo financiero existe la necesidad de contar con sistemas de alerta que permitan tomar oportunamente medidas correctivas con base en el comportamiento de las variables relevantes que pueden afectar el comportamiento del spread del crédito. Para contar con sistemas de alerta temprana que cumplan satisfactoriamente su función, se requieren explicaciones congruentes sobre el comportamiento dinámico del riesgo de crédito. El objetivo del presente trabajo es evaluar el impacto del riesgo sistemático, representado por el comportamiento de variables macroeconómicas locales, en la dinámica de la prima de riesgo del costo de la deuda de empresas mexicanas.

Este artículo contribuye a la literatura al presentar una forma novedosa de calcular la prima de riesgo o spread de crédito a partir de información financiera generada por la propia empresa. Al considerar el costo que la firma tiene por su deuda ${ }^{6} \mathrm{se}$ obtiene una medida más amplia del spread, lo cual diferencia este estudio de la literatura previa en donde se mide dicho spread con base en los rendimientos que pagan en el mercado de valores los bonos de las empresas. Asimismo, se ofrece evidencia empírica que permite relacionar el comportamiento de las primas de riesgo o spreads del costo de la deuda con el riesgo sistemático presente en el entorno económico nacional. Al respecto, es conveniente destacar que hasta donde sabemos, este estudio es el primero en su género. En última instancia, los hallazgos que se reportan más adelante confirman lo que han encontrado otros investigadores con respecto a variables económicas que pueden representar fuentes de riesgo sistemático en México.

En la siguiente sección se muestra la literatura relevante para este estudio. En la sección tres se describe la especificación del modelo econométrico mediante la cual intentamos explicar el comportamiento de la prima o spread que pagan importantes empresas mexicanas por concepto de su endeudamiento, con base en el desempeño de variables económicas. Los resultados del análisis econométrico

\footnotetext{
${ }^{5}$ Aspecto naturalmente relacionado con la eficiencia de la empresa y, en última instancia, con su rentabilidad. ${ }^{6}$ Específicamente aquella sujeta a un costo financiero activo, es decir, independiente del cumplimiento de la obligación en forma y tiempo.
} 
correspondiente se reportan en la sección cuatro. Finalmente, en la sección cinco, se presentan las conclusiones y recomendaciones.

\section{Revisión de literatura}

El riesgo de crédito representa una parte fundamental del riesgo financiero; incluso hay autores que aseguran que es el más importante en la economía al representar entre el 50\% y el 60\% del riesgo total (Hanson y Schuermann, 2006). En los últimos años la cantidad de estudios sobre el riesgo de crédito, específicamente sobre el comportamiento de la capacidad de pago en el transcurso del tiempo, se ha incrementado de forma importante; tal incremento se podría explicar a la luz de dos justificaciones: la económica y la regulatoria.

En cuanto a la justificación económica podemos mencionar a Wei (2003), Eiteman, Stonehill y Moffett (2001) y Moncarz, Moncarz, Cabello y Moncarz (2006), quienes documentan una serie de descalabros financieros tanto corporativos ${ }^{7}$ como soberanos. Altman y Saunders (1998) complementan esta línea de investigación con el análisis de otros factores de impacto económico entre los que destacan la tendencia hacia la desintermediación por parte de los deudores y el dramático crecimiento de los instrumentos off-balance sheet $t^{8}$ con exposición inherente al riesgo de incumplimiento. Complementando la idea, Partnoy (2001) señala que, en función de las pérdidas presentadas a partir de la ineficiente administración del riesgo de crédito, los inversionistas demandan investigación cada vez más sofisticada.

Por otro lado, los acuerdos de Basilea de 1988 sugerían, entre otras cosas, establecer como mínimo una proporción de capital para respaldar a los activos de los bancos originados por sus operaciones de crédito. Aunque tales acuerdos fueron uno de los mayores pasos para introducir la concepción de diferenciación en función de los riesgos del crédito; no resultaron ser una solución óptima porque no consideraban el tipo de activos, específicamente su riesgo (BIS, 1999). Así, en junio de 1999 se presentó la enmienda conocida como Basilea II, que introdujo un enfoque más realista en cuanto a los sistemas internos de calificación crediticia que considerara todos sus

\footnotetext{
${ }^{7}$ En forma obligada tendríamos que incluir lo sucedido en la economía de los Estados Unidos en los últimos meses, a partir de la crisis hipotecaria, donde las pérdidas acumuladas a septiembre de 2008 se estiman en 1.8 trillones de dólares, así como el contagio en los sistemas financieros de todo el mundo.

${ }^{8}$ Se refiere a los activos y actividades de endeudamiento o financiamiento que no están registrados en el balance general, como es el caso de las posiciones en instrumentos derivados (Altman y Saunders, 1998).
} 
aspectos: incumplimientos, migraciones, tasa de recuperación, spreads de crédito y concentraciones de riesgo (Altman y Onorato, 2004). Los lineamientos establecidos por Basilea II aún no estaban totalmente implementados a mediados de 2008, no obstante, pudieran haber quedado rebasados debido a la crisis financiero-económica que ha venido aquejando a los sistemas financieros del mundo.

La teoría financiera establece una relación directa entre riesgo y rendimiento, es decir, ante distintas alternativas de inversión, entre las que se incluya un activo libre de riesgo, un inversionista elegirá la opción más riesgosa únicamente ante la existencia de una prima que le compense adecuadamente por el riesgo adicional asumido. Las primas de riesgo se relacionan en gran medida con el riesgo de mercado y de crédito; tales primas fluctúan en función de los cambios en el mercado de capitales, que a su vez afectan el riesgo de crédito para todos los ratings (Crouhy, Galai y Mark, 2000; Delianedis y Geske, 2001; Farnsworth y Li, 2007). La teoría de la valuación de activos estudia cómo se fijan los precios y primas de riesgo partiendo del hecho de que el precio actual de un activo debe ser igual al pago esperado en el futuro, descontado por un factor que capture el efecto del paso del tiempo y el nivel de riesgo asociado con el flujo de efectivo esperado. Así, los activos más riesgosos deben ofrecer mayores primas de riesgo o spreads.

Entre los trabajos que utilizan el concepto de primas de riesgo en el contexto del riesgo de crédito tenemos a Altman y Saunders (1998), quienes encuentran que los cambios en la calidad crediticia afectan el costo de capital, los spreads de crédito, rendimientos de bonos, etc. Otros estudios que abordan la relación entre los spreads de mercado y la capacidad de pago son Cantor (2004); Hull, Predescu y White (2004); Bielecki y Rutkowski (2000); Sy (2003). La aplicación de spreads también se aprecia en el contexto específico de los mercados emergentes, como es el caso de Altman (2005), quien introduce un modelo de puntuación que integra factores entre los que destaca una medida de spread. En el mismo contexto de economías emergentes, Sy (2002) presenta evidencia de que los spreads de bonos incorporan las expectativas de cambios esperados en el rating, lo que a su vez funciona como un indicador del costo de capital al que aquellos pueden acceder en los mercados internacionales de capital. Delianedis y Geske (2003) aseguran que los precios de mercado anticipan cambios inminentes, meses antes que los refleje el rating. Partnoy (2001) complementa la idea al afirmar que el rating tiene alto valor de mercado, pero poco valor informativo y sugiere tomar como aproximación al riesgo de incumplimiento, el spread de crédito argumentando que contiene básicamente la misma información que el rating. 
A partir de la teoría de Harry Markowitz, presentada en 1952, se han realizado incontables esfuerzos por explicar, capturar, modelar y pronosticar los determinantes y efectos del riesgo sistemático o de mercado. De acuerdo con esa teoría, los precios de los activos financieros responden a eventos aleatorios que afectan las utilidades actuales y futuras de la empresa. En otras palabras, los cambios en las condiciones económicas y de mercado que se reflejan en cambios en tasas de interés, índices del mercado accionario, tipos de cambio, tasa de desempleo, etc., pueden afectar, y lo hacen, a la rentabilidad general de las empresas; así como también a la exposición de las contrapartes de un deudor y sus posibilidades de incumplimiento (Crouhy, Galai y Mark; 2000 y 2001). Desafortunadamente, uno de los principales problemas del estudio del riesgo de crédito es la falta de datos disponibles 9 (Gupton, Finger y Bhatia; 1997; BIS, 1999). Al respecto, Segoviano y Padilla (2006) aseguran que los intentos que han pretendido sortear tal dificultad han generado, a su vez, nuevas complicaciones como el uso de metodologías y análisis estadísticos que parten de supuestos poco realistas o simplemente ignoran los potenciales efectos de variables macroeconómicas.

La idea de considerar factores macroeconómicos en el estudio del riesgo de crédito no es nueva, en 1977 Charles Moyer ya aseguraba tal importancia. Posteriormente, otros estudiosos del tema han seguido reconociendo esa necesidad (Gilbert, Menon y Schwartz, 1990; Crouhy, Galai y Mark, 2001). La evidencia sugiere que la información histórica contiene fuerte poder explicativo del comportamiento de las probabilidades de incumplimiento, puesto que éste no es un evento repentino sino que es la conclusión de un proceso de deterioro. Se considera que el riesgo sistemático está definido por el estado general de la economía y se afirma que hay una relación importante entre las condiciones macroeconómicas, la calidad de los activos y los cambios en la calidad crediticia junto a la eventual posibilidad de quiebra financiera (Wendin y McNeil, 2006; Altman y Onorato, 2004). Tales reflexiones trascienden el ámbito corporativo puesto que, incluso en el terreno del riesgo de los países, se afirma que el rating soberano refleja la información contenida en indicadores macroeconómicos (Benell, Crabbe, Thomas, Gwilym, 2006).

La mayoría de los esfuerzos por explicar los efectos del riesgo sistemático sobre el precio de los activos ha sido realizado en el contexto internacional, con una mayor aplicación en la economía de los Estados Unidos, mientras que en México la

\footnotetext{
${ }^{9}$ Lo que explica que este tipo de estudios se realice en empresas listadas en bolsas organizadas garantizando, al menos en parte, la existencia de información financiera histórica.
} 
evidencia se reduce a unas cuantas investigaciones. Entre los estudios que abordan el tema en el contexto mexicano podemos mencionar a Luis de la Calle (1991), Nava (1996), Navarro y Santillán (2001), López y Vázquez (2002), Al-Shanfari (2003) y López (2006a y 2006b), quienes coinciden en que el riesgo sistemático se aprecia a través de la evolución de variables macroeconómicas. De la Calle (1991), Navarro y Santillán (2001), López y Vázquez (2002) y López (2006a) coinciden en señalar que el cambio en el nivel de precios constituye una dimensión importante del riesgo sistemático mexicano. De igual manera, el tipo de cambio es una variable importante según los hallazgos de Navarro y Santillán (2001), así como Doshi, Johnson, Ortiz y Soenen (2001). La tasa de interés también es un factor determinante, para el caso mexicano, según la evidencia empírica presentada por Navarro y Santillán (2001) y Al-Shanfari (2003).

De la Calle (1991), Navarro y Santillán (2001) y López (2006a y 2006b) confirman que un incremento inesperado en la oferta monetaria modifica el nivel de liquidez de los participantes del sistema financiero, lo que a su vez produce presiones a la baja en las tasas de interés dando impulso a la economía. Por otro lado, para López y Vázquez (2002) el nivel de actividad económica es otra variable importante en la valorización de los activos al representar la confianza de los distintos agentes económicos; ellos utilizan en su estudio como aproximación del nivel de actividad económica el Producto Interno Bruto (PIB). Otra medida importante del dinamismo de la economía es el comportamiento de su comercio con el exterior (López, 2006b). Al respecto, Navarro y Santillán (2001) encuentran en su estudio sobre el mercado mexicano una relación significativa de las exportaciones. No obstante, López (2006b) asegura que en virtud de que los efectos de las exportaciones y las importaciones sobre el riesgo sistemático han sido poco estudiados, es más conveniente estudiarlos por separado, pues podrían representar efectos distintos, aunque no necesariamente independientes. Finalmente, López (2006b) y López y Vázquez (2002) encuentran evidencia de la relevancia del impacto de las reservas internacionales sobre el riesgo sistemático de la economía mientras que De la Calle (1991), Navarro y Santillán (2001), López y Vázquez (2002) y López (2006a) confirman la importancia de los precios del petróleo en el mercado mexicano.

\section{Modelado del spread del costo de la deuda}

El spread de la deuda de cada empresa se calculó en tres etapas. En primera instancia se calculó el costo de la deuda, según se muestra a continuación: 


$$
\text { Costo de la deuda }=\frac{a}{b+c+d+e+f+g}
$$

donde:

$$
\begin{aligned}
& a=\text { Gastos financieros } \\
& b=\text { Deudas financieras de corto plazo } \\
& c=\text { Obligaciones negociables de corto plazo } \\
& d=\text { Otros créditos con costo de corto plazo } \\
& e=\text { Deudas financieras de largo plazo } \\
& f=\text { Obligaciones negociables de largo plazo } \\
& g=\text { Acreedores varios de largo plazo }
\end{aligned}
$$

En una segunda etapa y puesto que los elementos a que hace referencia la ecuación (1) son obtenidos de estados financieros trimestrales, y en virtud de que la tasa libre de riesgo utilizada ${ }^{10}$ para el cálculo de la prima está expresada en términos anuales, es necesario ajustar el costo de la deuda en los mismos términos, es decir, se calcula:

$$
\text { Costo anualizado de la deuda }=(1+\mathrm{i})^{4}-1
$$

Donde:

$$
\text { i = costo de la deuda obtenido a partir de la ecuación (1) }
$$

El spread se calculó como la diferencia entre el costo anualizado de la deuda, según la ecuación (1), menos la tasa libre de riesgo, a partir de lo cual se calculó su tasa de crecimiento de acuerdo con:

$$
y=\text { tasa de crecimiento del } \text { spread }=\frac{\text { spread }_{t}-\text { spread }_{t-1}}{\text { spread }_{t-1}}
$$

El modelo para estimar el efecto del riesgo sistemático sobre las tasas de crecimiento del spread o premio que paga por arriba de la tasa libre de riesgo cada una de las empresas debido a su deuda, incluyendo el término de perturbación aleatoria, se especifica como:

${ }^{10}$ CETES 28 días. Certificados de la Tesorería de la Federación. La serie utilizada se obtuvo de BANXICO. 


$$
y_{t}=\alpha+\sum_{i=0}^{4} \beta_{i} M 1_{t-i}+\sum_{i=0}^{4} \gamma_{i} P I B_{t-i}+\sum_{i=0}^{4} \delta_{i} \operatorname{RESINT}_{t-i}+\sum_{i=0}^{4} \phi_{i} T C_{t-i}+\sum_{i=0}^{4} \varphi_{i} I N F_{t-i}+\sum_{i=0}^{4} \lambda_{i} E X P_{t-i}+\sum_{i=0}^{4} \theta_{i} \operatorname{PETR}_{t-i}+\sum_{i=0}^{4} \psi_{i} I M P_{t-i}+\varepsilon_{t} .
$$

Este modelo dinámico se especifica con un intercepto y los valores contemporáneos y rezagados de los factores de riesgo presentados en la sección anterior. En virtud de que los estados financieros de las emisoras bajo estudio están disponibles en forma trimestral, se incluyen cuatro rezagos de los factores de riesgo para capturar sus efectos sobre la dinámica anual del spread del costo de la deuda de las empresas. La estimación por mínimos cuadrados ordinarios (MCO), que se presenta más adelante, se efectuó siguiendo el enfoque de lo general a lo específico, es decir, el propuesto por la metodología impulsada por la Escuela de Economía de Londres con base en el planteamiento de David Hendry. Partiendo de aceptar el hecho de que no se conoce el verdadero proceso que genera los datos observables, el procedimiento para la estimación de los parámetros relevantes puede definirse como un proceso de reducción secuencial del modelo original que inicia con una especificación tan amplia como sea posible (modelo no restringido), según lo permita la fundamentación teórica y el conocimiento previo que se tiene del proceso en cuestión. En cada fase de reducción se verifica, mediante pruebas de restricción, que las variables con coeficientes no significativos puedan retirarse de la especificación propuesta mejorando los criterios de selección del modelo (estadístico F y los criterios de Schwarz, Akaike y de Hannan-Quinn). El proceso continúa de esta forma hasta que todos los parámetros son significativos. El resultado de este procedimiento es que se obtiene un modelo (restringido o reducido) lo más parsimonioso posible y al mismo tiempo congruente, en términos de la fundamentación teórica ${ }^{11}$.

\section{Resultados de las estimaciones}

La información relativa a los estados financieros proviene de la base de datos Economática y se consideran únicamente aquellas emisoras que tengan al menos 60 observaciones trimestrales consecutivas ${ }^{12}$, es decir, el periodo de estudio abarca 15 años: de junio de 1992 a marzo de 2007. De acuerdo con este criterio, se analizan en total 28 emisoras de los siete sectores de la Bolsa Mexicana de Valores ${ }^{13}$ (BMV), según se muestra en el cuadro 1.

\footnotetext{
${ }^{11}$ Para mayores detalles puede consultarse Otero (1993) y Johnston y DiNardo (1997).

${ }^{12}$ Excepto BEVIDES, SAB y GMARTI con 2, 10 y 2 datos ausentes al inicio de la serie, respectivamente.

${ }^{13}$ Se considera la antigua clasificación y se excluye el sector financiero debido a la heterogeneidad en la información capturada por las razones financieras de empresas que pertenecen o no al sector financiero (Perry, Henderson y Cronan, 1984; Shumway, 2001, Carey y Hrycay, 2001; Livingston, Naranjo y Zhou, 2005).
} 


\section{Cuadro 1}

\section{Emisoras seleccionadas por sector de actividad económica}

\begin{tabular}{|c|c|c|c|}
\hline No. & Sector BMV & Empresa & Clave \\
\hline 1 & \multirow{5}{*}{ Varios } & Grupo Carso, S.A.B. de C.V. & GCARSO \\
\hline 2 & & Accel, S.A.B. de C.V. & ACCEL \\
\hline 3 & & Grupo Industrial Saltillo, S.A.B. de C.V. & GIISA \\
\hline 4 & & San Luis Corporación, S.A. de C.V. & SANLUIS \\
\hline 5 & & Alfa, S.A.B. de C.V. & ALFAA \\
\hline 6 & \multirow{2}{*}{ Extractiva } & Industrias Peñoles, S.A.B. de C.V. & PEÑOLES \\
\hline 7 & & Compañía Minera Autlán, S.A.B. de C.V. & AUTLAN \\
\hline 8 & \multirow{3}{*}{ Servicios } & Grupo Posadas, S.A. de C.V. & POSADA \\
\hline 9 & & Consorcio Aristos, S.A. de C.V. & ARISTOS \\
\hline 10 & & Real Turismo, S.A. de C.V. & REALTUR \\
\hline 11 & \multirow{6}{*}{ Transformación } & Grupo Bimbo, S.A.B. de C.V. & ВIмBO \\
\hline 12 & & Grupo la Moderna, S.A.B. de C.V. & GMODERN \\
\hline 13 & & Ekco, S.A.B. & EKCO \\
\hline 14 & & Altos Hornos de México, S.A. de C.V. & AHMSA \\
\hline 15 & & Vitro, S.A.B. de C.V. & VITRO \\
\hline 16 & & Gruma, S.A.B. de C.V. & GRUMA \\
\hline 17 & \multirow{3}{*}{ Construcción } & Grupo Cementos de Chihuahua, S.A.B. de C.V. & GCC \\
\hline 18 & & Empresas ICA, S.A.B. de C.V. & ICA \\
\hline 19 & & CEMEX, S.A.B. de C.V. & CEMEX \\
\hline 20 & \multirow{6}{*}{ Comercial } & Grupo Gigante, S.A.B. de C.V. & GIGANTE \\
\hline 21 & & Farmacias Benavides, S.A.B. de C.V. & BEVIDES \\
\hline 22 & & Controladora Comercial Mexicana, S.A.B. de C.V. & COMERCI \\
\hline 23 & & Grupo Casa Saba, S.A.B. de C.V. & SAB \\
\hline 24 & & Grupo Martí, S.A.B. & GMARTI \\
\hline 25 & & El Puerto de Liverpool, S.A.B. de C.V. & LIVERPOL \\
\hline 26 & \multirow{3}{*}{$\begin{array}{l}\text { Comunicaciones y } \\
\text { transportes }\end{array}$} & Teléfonos de México, S.A.B. de C.V. & TELMEX \\
\hline 27 & & Grupo тмм, S.A. & TMM \\
\hline 28 & & Grupo Televisa, S.A. & TELEVISA \\
\hline
\end{tabular}

En el cuadro 2 se enuncian los factores de riesgo seleccionados para estimar el modelo de la ecuación (4), así como la fuente de los datos base. Los factores de riesgo se obtuvieron al estimar las tasas de crecimiento de dichos datos siguiendo el procedimiento descrito en la ecuación (3). 


\section{Cuadro 2}

\section{Factores de riesgo y fuentes de información}

\begin{tabular}{|c|c|c|}
\hline Factor de riesgo & Nomenclatura & Fuente de los datos base \\
\hline Tasa de inflación & INF & BANXICO \\
\hline Tasa de apreciación del dólar frente al peso & TC & BANXICO \\
\hline Crecimiento de la oferta monetaria & MI & BANXICO \\
\hline Crecimiento del PIB & $\mathrm{PIB}$ & INEGI \\
\hline Crecimiento de las exportaciones totales & EXP & SHCP \\
\hline Crecimiento de las importaciones totales & IMP & SHCP \\
\hline Crecimiento de las reservas internacionales & RESINT & BANXICO \\
\hline Crecimiento de los precios del petróleo ${ }^{14}$ & PETR & ECONOMÁTICA \\
\hline
\end{tabular}

Dado que el desempeño de la economía mexicana ha demostrado históricamente una gran dependencia de los Estados Unidos, parece tentador considerar variables relevantes de esa economía para incluirlas en el análisis. Sin embargo, nuestro objetivo es recabar evidencia del efecto de variables económicas locales como determinantes de las primas de riesgo de crédito en México. Por otra parte, el impacto de variables económicas relevantes de Estados Unidos, al menos en su mayor parte, ya se refleja en las variables utilizadas en este estudio, principalmente a través el PIB, los precios y las exportaciones. Como ejemplo, tomando del primer trimestre de 1992 al cuarto trimestre del 2007, se encuentra una correlación de 0.97 entre los productos internos brutos de ambos países y de la misma magnitud para sus niveles de precios al consumidor. Otra variable utilizada en este estudio, que también captura efectos directos de la evolución de la economía estadounidense es indudablemente el comportamiento de las exportaciones mexicanas hacia ese país. Según BANXICO, las exportaciones promedio de México hacia EUA, como porcentaje del PIB, fueron de $86.3 \%$ entre 2003 y 2007; en 2008 dicho porcentaje fue de $80.1 \%$, el valor más bajo del periodo. También las importaciones de EUA hacia México son importantes, pues de 2003 a 2008 del total de importaciones mexicanas el $61.8 \%, 56.3 \%, 53.4 \%, 51 \%, 50 \%$ y $49 \%$ provienen de aquel país.

En el cuadro 3 se presentan los resultados de las estimaciones, las cuales se realizaron mediante el paquete econométrico Gretl, versión 1.8. Por motivos de espacio, dicho cuadro muestra únicamente las estimaciones obtenidas mediante el proceso descrito en la sección anterior, es decir, los resultados corresponden únicamente a los modelos restringidos.

\footnotetext{
${ }^{14}$ MAYA: Mezcla de petróleo crudo pesado que se produce en México con fines de exportación, el cual tiene una densidad alrededor de 22 API.
} 


\section{Cuadro 3}

Estimaciones del modelo del comportamiento del spread de deuda

\begin{tabular}{|c|c|c|c|c|c|c|c|}
\hline VARIABLES & ACCEL & AHMSA & ALFA & ARISTOS & AUTLAN & BEVIDES & BIMBO \\
\hline Constante & ns & ns & ns & $-8.53 *$ & $12.69 * * *$ & $11.51 *$ & ns \\
\hline M1 & ns & ns & $-26.19 *$ & $39.78 * * *$ & ns & ns & ns \\
\hline$M 1_{t-1}$ & ns & $\mathrm{ns}$ & ns & $41.76 * *$ & ns & ns & $\mathrm{ns}$ \\
\hline $\mathrm{M} 1_{\mathrm{t}-2}$ & $16.95 * * *$ & $11.72 * *$ & $36.41 * * *$ & $20.17 * *$ & $\mathrm{~ns}$ & $-20.29 *$ & $15.98 * *$ \\
\hline $\mathrm{M} 1_{\mathrm{t}-3}$ & $\mathrm{~ns}$ & $5.38 * * *$ & $69.31 * * *$ & $14.7 * *$ & $-19.92 * * *$ & ns & $8.18 *$ \\
\hline $\mathrm{M} 1_{\mathrm{t}-4}$ & $\mathrm{~ns}$ & ns & ns & ns & ns & ns & $-24.98 * *$ \\
\hline PIB & $\mathrm{ns}$ & $\mathrm{ns}$ & $235.5 * * *$ & $-78.38 * *$ & 94.30 *** & $\mathrm{ns}$ & $\mathrm{ns}$ \\
\hline $\mathrm{PIB}_{\mathrm{t}-1}$ & $-29.93 * *$ & $\mathrm{~ns}$ & $-115.24 * *$ & $-89.5 * *$ & $201.49 * * *$ & $\mathrm{~ns}$ & $\mathrm{~ns}$ \\
\hline $\mathrm{PIB}_{\mathrm{t}-2}$ & $-50.64 * * *$ & $-24.09 * *$ & $-142.8 * * *$ & ns & $142.78 * * *$ & ns & $-45.17 * * *$ \\
\hline $\mathrm{PIB}_{\mathrm{t}-3}$ & ns & $-15.99 * *$ & $-133.62 * * *$ & ns & $\mathrm{ns}$ & $-64.15 *$ & $30.23 *$ \\
\hline $\mathrm{PIB}_{\mathrm{t}-4}$ & $\mathrm{~ns}$ & ns & $-159.74 * * *$ & ns & $-58.89 * * *$ & ns & $107.51 * *$ \\
\hline RESINT & ns & $-6.78 * * *$ & $33.06 * * *$ & $-5.33 *$ & $-8.7 *$ & ns & $-6.91 * *$ \\
\hline RESINT $_{t-1}$ & ns & ns & ns & ns & $-14.55 * * *$ & ns & ns \\
\hline RESINT $_{\mathrm{t}-2}$ & $\mathrm{~ns}$ & ns & $-25.16 * * *$ & ns & ns & $\mathrm{ns}$ & $7.73 * *$ \\
\hline RESINT $_{t-3}$ & $4.25 *$ & $-1.38 *$ & ns & $\mathrm{ns}$ & $9.7 * * *$ & ns & ns \\
\hline RESINT $_{\mathrm{t}-4}$ & $-6.3 * * *$ & ns & ns & $\mathrm{ns}$ & $13.08 * * *$ & ns & ns \\
\hline TC & ns & ns & ns & $\mathrm{ns}$ & $68.79 * * *$ & $\mathrm{~ns}$ & ns \\
\hline $\mathrm{TC}_{\mathrm{t}-1}$ & $-5.78 * * *$ & ns & $-19.63 * *$ & ns & $31.66 * * *$ & $-1.33 *$ & $33.45 * *$ \\
\hline $\mathrm{TC}_{\mathrm{t}-2}$ & ns & ns & $22.47 *$ & $-28.6 * *$ & ns & $-9.67 *$ & $6.06 * *$ \\
\hline $\mathrm{TC}_{\mathrm{t}-3}$ & $\mathrm{~ns}$ & $\mathrm{~ns}$ & ns & ns & ns & ns & $10.79 *$ \\
\hline $\mathrm{TC}_{\mathrm{t}-4}$ & ns & ns & $-43.08 * *$ & ns & $-9.38 * * *$ & $\mathrm{~ns}$ & $33.33 * *$ \\
\hline INF & $\mathrm{ns}$ & $-55.16 *$ & $239.33 * *$ & $-47.51 * *$ & $-111.89 * * *$ & $\mathrm{~ns}$ & $-153.04 * * *$ \\
\hline $\mathrm{INF}_{\mathrm{t}-1}$ & ns & ns & ns & ns & $-231.43 * * *$ & ns & ns \\
\hline $\mathrm{INF}_{\mathrm{t}-2}$ & $\mathrm{~ns}$ & $\mathrm{~ns}$ & $-83.76^{* *}$ & ns & $178.19 * * *$ & $\mathrm{~ns}$ & $-56.29 *$ \\
\hline $\mathrm{INF}_{\mathrm{t}-3}$ & $\mathrm{~ns}$ & $\mathrm{~ns}$ & ns & $-171.98 * * *$ & $211.76 * * *$ & $\mathrm{~ns}$ & ns \\
\hline $\mathrm{INF}_{\mathrm{t}-4}$ & $\mathrm{~ns}$ & $\mathrm{~ns}$ & $-118.38 * * *$ & $52.19 * *$ & $-123.27 * * *$ & $\mathrm{~ns}$ & 86.63 *** \\
\hline EXP & $\mathrm{ns}$ & $\mathrm{ns}$ & ns & ns & $-8.54 * *$ & $\mathrm{~ns}$ & $-3.23 * * *$ \\
\hline $\mathrm{EXP}_{\mathrm{t}-1}$ & $\mathrm{~ns}$ & $\mathrm{~ns}$ & ns & ns & ns & $\mathrm{ns}$ & $-7.97 *$ \\
\hline $\mathrm{EXP}_{\mathrm{t}-2}$ & ns & $\mathrm{ns}$ & $-11.62 * *$ & $9.53 * *$ & $18.16 * * *$ & $\mathrm{~ns}$ & ns \\
\hline $\mathrm{EXP}_{\mathrm{t}-3}$ & $-3.85 * * *$ & $\mathrm{~ns}$ & ns & $3.68 * *$ & $2.5 * *$ & $\mathrm{~ns}$ & $-4.03 * *$ \\
\hline $\mathrm{EXP}_{\mathrm{t}-4}$ & $\mathrm{~ns}$ & ns & $18.26 * *$ & ns & ns & ns & $-13.37 * *$ \\
\hline PETR & ns & $\mathrm{ns}$ & ns & ns & $-11.75 * * *$ & $\mathrm{~ns}$ & $\mathrm{~ns}$ \\
\hline PETR $_{t-1}$ & $3.52 * * *$ & ns & ns & $2.57 * * *$ & $-2.67 * * *$ & ns & ns \\
\hline PETR $_{\mathrm{t}-2}$ & ns & ns & $3.67 * * *$ & $2.57 * *$ & ns & ns & ns \\
\hline PETR $_{t-3}$ & $1.58 * *$ & ns & ns & $-2.07 *$ & ns & ns & $2.84 * * *$ \\
\hline PETR $_{\mathrm{t}-4}$ & $-0.97 *$ & ns & ns & $-2.0 * * *$ & $-2.14 * * *$ & ns & ns \\
\hline IMP & ns & $6.28 * *$ & $-23.12 * *$ & $9.05 * *$ & $-33.07 * * *$ & ns & $13.31 * *$ \\
\hline $\mathrm{IMP}_{\mathrm{t}-1}$ & ns & $7.4 * *$ & ns & $\mathrm{ns}$ & ns & ns & ns \\
\hline $\mathrm{IMP}_{\mathrm{t}-2}$ & ns & $5.87 * *$ & $\mathrm{~ns}$ & $\mathrm{~ns}$ & $-47.0 * * *$ & $14.5 *$ & ns \\
\hline $\mathrm{IMP}_{\mathrm{t}-3}$ & $8.68 * *$ & $2.68 *$ & Ns & ns & $-26.41 * * *$ & ns & ns \\
\hline $\mathrm{IMP}_{\mathrm{t}-4}$ & $3.28 *$ & ns & Ns & $15.05 * * *$ & ns & ns & ns \\
\hline $\mathrm{R}^{2}$ ajustada & 0.30 & 0.14 & 0.52 & 0.29 & 0.84 & 0.08 & 0.82 \\
\hline Durbin-Watson & 2.12 & 2.15 & 2.52 & 2.41 & 2.41 & 1.96 & 2.73 \\
\hline C. de Schwarz & 459.57 & 435.06 & 534.62 & 471.97 & 470.13 & 496.97 & 456.83 \\
\hline
\end{tabular}




\section{Continuación cuadro 3}

\begin{tabular}{|c|c|c|c|c|c|c|c|}
\hline VARIABLES & CEMEX & COMERCI & ECKO & GCARSO & GCC & GIGANTE & GIISA \\
\hline Constante & $-110.22 *$ & $-5.45 * *$ & $4.21 * * *$ & $7.77 * * *$ & ns & ns & ns \\
\hline M1 & $269.74 *$ & $28.98 * * *$ & $-2.06 * *$ & $-6.32 * * *$ & $15.05 * * *$ & $-8.03 * * *$ & $-22.94 * * *$ \\
\hline $\mathrm{M} 1_{\mathrm{t}-1}$ & ns & $-4.67 *$ & $-9.52 * * *$ & $-17.95 * * *$ & ns & ns & ns \\
\hline $\mathrm{M} 1_{\mathrm{t}-2}$ & $194.79 *$ & $19.07 * * *$ & ns & $-7.86 * * *$ & $19.97 * * *$ & ns & $14.37 * * *$ \\
\hline $\mathrm{M} 1_{\mathrm{t}-3}$ & ns & $10.37 * * *$ & ns & $-7.39 * * *$ & $11.55 * * *$ & ns & $14.71 * * *$ \\
\hline $\mathrm{M} 1_{\mathrm{t}-4}$ & $-225.93 *$ & $-19.05 * * *$ & ns & ns & ns & ns & ns \\
\hline PIB & $395.4 *$ & $38.57 * *$ & ns & $18.49 * * *$ & ns & ns & $61.0 * * *$ \\
\hline $\mathrm{PIB}_{\mathrm{t}-1}$ & $1371.5 * *$ & $71.12 * * *$ & $\mathrm{~ns}$ & $29.87 * * *$ & ns & ns & $-55.87 * * *$ \\
\hline $\mathrm{PIB}_{\mathrm{t}-2}$ & $1030.92 * *$ & ns & $-15.2 * * *$ & $9.92 *$ & ns & $-21.03 * * *$ & $-69.17 * * *$ \\
\hline $\mathrm{PIB}_{\mathrm{t}-3}$ & $1636.01 * *$ & $37.82 * * *$ & $-9.64 * * *$ & ns & ns & $\mathrm{ns}$ & ns \\
\hline $\mathrm{PIB}_{\mathrm{t}-4}$ & $1163.99 * *$ & $44.4 * * *$ & ns & ns & $-31.55 * * *$ & $23.02 * * *$ & ns \\
\hline RESINT & $-99.33 *$ & $-5.93 * *$ & $1.37 * *$ & ns & $3.63 * *$ & ns & ns \\
\hline RESINT $_{t-1}$ & ns & ns & $2.23 *$ & $4.11 * * *$ & ns & $8.58 *$ & ns \\
\hline RESINT $_{t-2}$ & ns & $-3.74 *$ & $-1.58 * * *$ & ns & $-8.35 * * *$ & $8.52 * * *$ & ns \\
\hline RESINT $_{t-3}$ & ns & ns & $-1.57 * * *$ & ns & $6.81 * * *$ & ns & $7.32 * *$ \\
\hline RESINT $_{t-4}$ & $\mathrm{~ns}$ & $-2.5 * *$ & $3.29 * * *$ & ns & ns & ns & $-4.29 * * *$ \\
\hline TC & $263.95 *$ & $4.79 * * *$ & $-3.76 * *$ & $-5.96 * * *$ & $-22.6 * * *$ & $-11.99 * * *$ & ns \\
\hline $\mathrm{TC}_{\mathrm{t}-1}$ & $155.01 *$ & $6.54 * * *$ & ns & ns & $14.54 * * *$ & $-15.92 * * *$ & $-30.05 * * *$ \\
\hline $\mathrm{TC}_{\mathrm{t}-2}$ & $207.93 * *$ & $19.02 * * *$ & $-3.4 * * *$ & ns & ns & $-8.41 * * *$ & $6.65 *$ \\
\hline $\mathrm{TC}_{\mathrm{t}-3}$ & $243.74 *$ & $14.87 * * *$ & ns & ns & $-4.06 *$ & $8.1 * *$ & ns \\
\hline $\mathrm{TC}_{\mathrm{t}-4}$ & $142.21 *$ & $6.13 * * *$ & ns & $3.68 * * *$ & ns & ns & $-22.51 * * *$ \\
\hline INF & ns & $-46.04 * *$ & ns & $34.94 * * *$ & 106.14 **** & $118.44 * * *$ & ns \\
\hline $\mathrm{INF}_{\mathrm{t}-1}$ & ns & ns & ns & ns & ns & ns & ns \\
\hline $\mathrm{INF}_{\mathrm{t}-2}$ & ns & ns & $16.27 * * *$ & $67.0 * * *$ & $-82.45 * * *$ & ns & ns \\
\hline $\mathrm{INF}_{\mathrm{t}-3}$ & $-565.94 *$ & ns & ns & ns & $41.31 * * *$ & $-72.81 * *$ & $79.94 * * *$ \\
\hline $\mathrm{INF}_{\mathrm{t}-4}$ & ns & $-23.81 * *$ & ns & $-14.53 * * *$ & $-75.85 * * *$ & ns & $-44.25 * *$ \\
\hline EXP & $-83.79 *$ & ns & $2.4 * * *$ & $3.97 * * *$ & 8.55 *** & ns & $1.31 * *$ \\
\hline $\mathrm{EXP}_{\mathrm{t}-1}$ & ns & ns & ns & ns & $-8.13 * * *$ & $4.43 * *$ & $5.59 *$ \\
\hline $\mathrm{EXP}_{\mathrm{t}-2}$ & ns & $-3.97 * *$ & ns & ns & $1.49 * *$ & ns & $\mathrm{ns}$ \\
\hline $\mathrm{EXP}_{\mathrm{t}-3}$ & ns & $-5.27 * * *$ & ns & $-0.59 * *$ & ns & $-4.54 * *$ & ns \\
\hline $\mathrm{EXP}_{\mathrm{t}-4}$ & ns & ns & ns & ns & ns & ns & $8.38 * * *$ \\
\hline PETR & ns & ns & ns & ns & ns & ns & $-2.05 * *$ \\
\hline PETR $_{t-1}$ & ns & $-1.09 * *$ & $\mathrm{~ns}$ & ns & ns & ns & ns \\
\hline PETR $_{t-2}$ & ns & ns & $-0.54 * * *$ & $-1.42 * * *$ & ns & $1.57 * *$ & $1.56 * *$ \\
\hline PETR $_{t-3}$ & ns & ns & ns & ns & ns & $0.82 * * *$ & $1.15 *$ \\
\hline PETR $_{t-4}$ & ns & ns & $0.69 * * *$ & $0.9 * * *$ & ns & ns & $-2.7 * * *$ \\
\hline IMP & $\mathrm{ns}$ & ns & $-5.39 * * *$ & $-10.88 * * *$ & $-11.5 * *$ & $8.99 * *$ & ns \\
\hline $\mathrm{IMP}_{\mathrm{t}-1}$ & $-178.39 *$ & ns & $-2.43 * * *$ & $-6.63 * * *$ & ns & $-6.5 * *$ & $23.79 * * *$ \\
\hline $\mathrm{IMP}_{\mathrm{t}-2}$ & $-216.6 * *$ & ns & $3.79 * *$ & $-4.3 * * *$ & $\mathrm{~ns}$ & ns & $-14.12 * *$ \\
\hline $\mathrm{IMP}_{\mathrm{t}-3}$ & $-322.11 * *$ & $\mathrm{~ns}$ & ns & $-4.95 * * *$ & $10.18 * *$ & ns & $-12.89 * * *$ \\
\hline $\mathrm{IMP}_{\mathrm{t}-4}$ & $-141.62 *$ & $-4.79 * *$ & $-1.47 * *$ & $-8.78 * * *$ & ns & ns & ns \\
\hline $\mathrm{R}^{2}$ ajustada & 0.23 & 0.67 & 0.62 & 0.72 & 0.37 & 0.46 & 0.57 \\
\hline Durbin-Watson & 2.03 & 1.76 & 2.67 & 1.82 & 2.28 & 2.24 & 2.60 \\
\hline C. de Schwarz & 720.01 & 395.01 & 304.71 & 333.65 & 439.25 & 404.99 & 430.51 \\
\hline
\end{tabular}




\section{Continuación cuadro 3}

\begin{tabular}{|c|c|c|c|c|c|c|c|}
\hline VARIABLES & GMARTI & GMODERN & GRUMA & ICA & LIVERPOL & PEÑOLES & POSADA \\
\hline Constante & ns & $\mathrm{ns}$ & $-7.18 * * *$ & $5.20 * * *$ & $4.58 *$ & $5.68 * * *$ & ns \\
\hline M1 & $\mathrm{ns}$ & $-86.84 * * *$ & $\mathrm{Ns}$ & ns & $-37.72 * * *$ & ns & $\mathrm{ns}$ \\
\hline $\mathrm{M} 1_{\mathrm{t}-1}$ & $10.2 * * *$ & $-17.88 *$ & Ns & $-12.16 * * *$ & $-22.28 * * *$ & $\mathrm{~ns}$ & $\mathrm{~ns}$ \\
\hline $\mathrm{M} 1_{\mathrm{t}-2}$ & $6.04 * *$ & $-27.56 *$ & $25.33 * * *$ & ns & $18.55 * *$ & $\mathrm{~ns}$ & $\mathrm{~ns}$ \\
\hline $\mathrm{M} 1_{\mathrm{t}-3}$ & $4.86 * *$ & ns & $15.71 * * *$ & $\mathrm{~ns}$ & ns & ns & $\mathrm{ns}$ \\
\hline $\mathrm{M} 1_{\mathrm{t}-4}$ & $-8.37 * *$ & $43.19 * *$ & ns & ns & $41.56^{* * * *}$ & $-14.79 * *$ & ns \\
\hline PIB & $-28.67 * * *$ & $-301.23 * * *$ & ns & $\mathrm{ns}$ & ns & ns & $-204.33 * *$ \\
\hline $\mathrm{PIB}_{\mathrm{t}-1}$ & ns & $-214.28 * * *$ & ns & $17.26 *$ & ns & $\mathrm{ns}$ & ns \\
\hline $\mathrm{PIB}_{\mathrm{t}-2}$ & $\mathrm{~ns}$ & $-175.95 * *$ & $\mathrm{~ns}$ & $\mathrm{~ns}$ & $-112.2 * * *$ & ns & $\mathrm{ns}$ \\
\hline $\mathrm{PIB}_{\mathrm{t}-3}$ & ns & $-368.91 * * *$ & $\mathrm{~ns}$ & ns & ns & $14.58 *$ & $\mathrm{~ns}$ \\
\hline $\mathrm{PIB}_{\mathrm{t}-4}$ & $59.45 * * *$ & ns & ns & ns & ns & $55.05 * *$ & $131.02 * *$ \\
\hline RESINT & $-4.95 * * *$ & ns & $13.18 * *$ & $\mathrm{~ns}$ & ns & ns & ns \\
\hline RESINT $_{\mathrm{t}-1}$ & ns & ns & ns & $\mathrm{ns}$ & $6.5 *$ & ns & $\mathrm{ns}$ \\
\hline RESINT $_{\mathrm{t}-2}$ & $4.2 * *$ & $35.57 * * *$ & $-6.26 * *$ & $\mathrm{~ns}$ & $8.32 * * *$ & $3.86 * * *$ & $\mathrm{~ns}$ \\
\hline RESINT $_{\mathrm{t}-3}$ & ns & $73.51 * * *$ & $4.94 * *$ & $-1.63 * *$ & $7.06 * *$ & ns & $\mathrm{ns}$ \\
\hline RESINT $_{\mathrm{t}-4}$ & $\mathrm{~ns}$ & $9.27 *$ & $8.91 * * *$ & ns & ns & $\mathrm{ns}$ & $\mathrm{ns}$ \\
\hline TC & $\mathrm{ns}$ & $31.2 * *$ & $-52.64 * * *$ & ns & $-24.09 * * *$ & $\mathrm{~ns}$ & $\mathrm{~ns}$ \\
\hline $\mathrm{TC}_{\mathrm{t}-1}$ & $9.66 * * *$ & $-65.31 * *$ & $-19.05 * *$ & ns & ns & $5.63 * * *$ & ns \\
\hline $\mathrm{TC}_{\mathrm{t}-2}$ & $5.18 * * *$ & $-128.26 * * *$ & $\mathrm{~ns}$ & $\mathrm{~ns}$ & ns & ns & ns \\
\hline $\mathrm{TC}_{\mathrm{t}-3}$ & $8.19 * *$ & $-272.96 * * *$ & ns & $0.5 * *$ & ns & $9.43 * * *$ & $\mathrm{~ns}$ \\
\hline $\mathrm{TC}_{\mathrm{t}-4}$ & $17.02 * * *$ & ns & ns & $3.89 * *$ & ns & $15.46 * *$ & $100.47 *$ \\
\hline INF & $-43.04 *$ & $\mathrm{~ns}$ & $\mathrm{~ns}$ & $11.5 * * *$ & $-158.67 * * *$ & $-67.95 * *$ & $-118.64 * *$ \\
\hline $\mathrm{INF}_{\mathrm{t}-1}$ & $-56.13 * * *$ & $932.2 * * *$ & ns & ns & ns & $-40.66 * * *$ & ns \\
\hline $\mathrm{INF}_{\mathrm{t}-2}$ & ns & $-430.64 * * *$ & $-169.32 * * *$ & ns & ns & $\mathrm{ns}$ & $\mathrm{ns}$ \\
\hline $\mathrm{INF}_{\mathrm{t}-3}$ & ns & $491.31 * * *$ & 97.78 *** & ns & 109.11 *** & ns & $\mathrm{ns}$ \\
\hline $\mathrm{INF}_{\mathrm{t}-4}$ & $32.51 * *$ & $-559.08 * * *$ & $-56.1 * * *$ & ns & ns & $41.01 * * *$ & ns \\
\hline EXP & $\mathrm{ns}$ & $-16.03 * *$ & 17.89 *** & ns & $10.91 * * *$ & $1.14 * *$ & ns \\
\hline $\mathrm{EXP}_{\mathrm{t}-1}$ & ns & $-13.68 *$ & $5.75 *$ & $\mathrm{~ns}$ & $\mathrm{~ns}$ & ns & ns \\
\hline $\mathrm{EXP}_{\mathrm{t}-2}$ & ns & $33.97 * * *$ & $2.05 *$ & ns & ns & ns & ns \\
\hline $\mathrm{EXP}_{\mathrm{t}-3}$ & $-2.59 * *$ & $67.12 * * *$ & ns & ns & ns & $-3.04 * *$ & $2.44 * * *$ \\
\hline $\mathrm{EXP}_{\mathrm{t}-4}$ & $-6.21 * * *$ & $-21.95 * * *$ & ns & $\mathrm{ns}$ & $-2.49 * * *$ & $-5.77 * *$ & $-34.42 *$ \\
\hline PETR & $\mathrm{ns}$ & $6.49 * * *$ & ns & ns & ns & ns & ns \\
\hline PETR $_{t-1}$ & ns & ns & ns & $-0.7 * * *$ & ns & ns & ns \\
\hline PETR $_{t-2}$ & ns & $-3.74 * *$ & $1.99 * *$ & $\mathrm{~ns}$ & $\mathrm{~ns}$ & $\mathrm{~ns}$ & $\mathrm{~ns}$ \\
\hline PETR $_{t-3}$ & ns & $8.88 * * *$ & $1.46 *$ & $\mathrm{~ns}$ & $2.98 * *$ & $\mathrm{~ns}$ & $\mathrm{~ns}$ \\
\hline PETR $_{\mathrm{t}-4}$ & $-0.5 * *$ & $11.48 * * *$ & ns & ns & ns & $\mathrm{ns}$ & $\mathrm{ns}$ \\
\hline IMP & $6.54 * * *$ & ns & ns & $-1.99 * * *$ & ns & $\mathrm{ns}$ & $\mathrm{ns}$ \\
\hline $\mathrm{IMP}_{\mathrm{t}-1}$ & $-5.29 * *$ & $75.39 * * *$ & $\mathrm{~ns}$ & ns & $14.88 * * *$ & ns & ns \\
\hline $\mathrm{IMP}_{\mathrm{t}-2}$ & ns & ns & ns & ns & ns & $4.56 * * *$ & $44.81 * * *$ \\
\hline $\mathrm{IMP}_{\mathrm{t}-3}$ & $\mathrm{~ns}$ & $49.52 * * *$ & $11.25 * *$ & ns & $-14.82 * * *$ & ns & ns \\
\hline $\mathrm{IMP}_{\mathrm{t}-4}$ & ns & ns & ns & $-4.97 * *$ & ns & ns & ns \\
\hline $\mathrm{R}^{2}$ ajustada & 0.42 & 0.63 & 0.52 & 0.31 & 0.45 & 0.43 & 0.46 \\
\hline Durbin-Watson & 2.49 & 2.08 & 1.93 & 2.04 & 2.28 & 2.21 & 1.89 \\
\hline C. de Schwarz & 340.88 & 581.50 & 460.51 & 347.34 & 469.84 & 380.22 & 627.66 \\
\hline
\end{tabular}




\section{Continuación cuadro 3}

\begin{tabular}{|c|c|c|c|c|c|c|c|}
\hline VARIABLES & REALTUR & SAB & SANLUIS & TELEVISA & TELMEX & TMM & VITRO \\
\hline Constante & Ns & $-2578.3 * * *$ & $2.47 * * *$ & $-82.73 * *$ & ns & $1.39 * *$ & $5.55 * *$ \\
\hline M1 & Ns & $3897.56 * * *$ & ns & $204.35 * *$ & $5.37 * * *$ & ns & ns \\
\hline $\mathrm{M} 1_{\mathrm{t}-1}$ & $-136.59 * * *$ & $2772.66 * * *$ & $\mathrm{~ns}$ & $\mathrm{~ns}$ & $-2.64 *$ & $-2.27 * * *$ & ns \\
\hline $\mathrm{M} 1_{\mathrm{t}-2}$ & 62.39 **** & $2236.9 * * *$ & $\mathrm{~ns}$ & $171.51 * *$ & $10.86 * * *$ & $\mathrm{~ns}$ & $15.92 * * *$ \\
\hline $\mathrm{M} 1_{\mathrm{t}-3}$ & $-45.79 * *$ & $3319.06 * * *$ & $\mathrm{~ns}$ & ns & $6.59 * * *$ & $\mathrm{~ns}$ & ns \\
\hline$M 1_{t-4}$ & $-214.84 * * *$ & $1875.54 * * *$ & ns & $-164.39 * *$ & $-4.69 * * *$ & $-2.49 * * *$ & $\mathrm{~ns}$ \\
\hline PIB & $561.76 * * *$ & $5856.87 * *$ & ns & $461.63 * *$ & ns & ns & $-68.85 * * *$ \\
\hline $\mathrm{PIB}_{\mathrm{t}-1}$ & Ns & $8015.55 * * *$ & $26.25 * * *$ & $1289.25 * *$ & $\mathrm{~ns}$ & ns & $-26.28 * *$ \\
\hline $\mathrm{PIB}_{\mathrm{t}-2}$ & $-287.05 * * *$ & $11520.8 * * *$ & $36.08 * * *$ & $1007.26 * *$ & $-14.41 * * *$ & ns & $\mathrm{ns}$ \\
\hline $\mathrm{PIB}_{\mathrm{t}-3}$ & $585.73 * * *$ & ns & $14.58 * * *$ & $1253.73 * *$ & ns & ns & $\mathrm{ns}$ \\
\hline $\mathrm{PIB}_{\mathrm{t}-4}$ & $258.17 * * *$ & $-14558 * * *$ & ns & $599.46 * *$ & $14.2 * *$ & ns & $25.86 * *$ \\
\hline RESINT & $19.20 *$ & $1521.38 * * *$ & ns & $-84.83 * *$ & ns & ns & $-6.72 * * *$ \\
\hline RESINT $_{t-1}$ & $\mathrm{Ns}$ & ns & $-3.36^{*}$ & ns & ns & ns & ns \\
\hline RESINT $_{\mathrm{t}-2}$ & Ns & $-1263.27 * * *$ & ns & ns & $-3.59 * * *$ & $\mathrm{~ns}$ & $-4.84 *$ \\
\hline RESINT $_{\mathrm{t}-3}$ & $78.47 * * *$ & $\mathrm{~ns}$ & ns & $\mathrm{ns}$ & $-0.82 *$ & $2.09 * *$ & $-5.57 * *$ \\
\hline RESINT $_{\mathrm{t}-4}$ & $-53.99 * * *$ & $2916.26 * * *$ & $5.69 * * *$ & ns & ns & $0.76 *$ & $\mathrm{~ns}$ \\
\hline $\mathrm{TC}$ & $-254.18 * * *$ & $-2053.77 * * *$ & $-6.95 * * *$ & $315.64 * *$ & $\mathrm{~ns}$ & ns & $\mathrm{ns}$ \\
\hline $\mathrm{TC}_{\mathrm{t}-1}$ & $134.16 * * *$ & $2222.78 * * *$ & $9.05 * * *$ & $152.55 * *$ & $\mathrm{~ns}$ & $\mathrm{~ns}$ & $\mathrm{~ns}$ \\
\hline $\mathrm{TC}_{\mathrm{t}-2}$ & $271.99 * * * *$ & $-1045.07 * *$ & ns & $234.38 * *$ & $8.27 * * *$ & $-2.46^{* * * *}$ & ns \\
\hline $\mathrm{TC}_{\mathrm{t}-3}$ & Ns & ns & $5.74 * * *$ & $385.6 * *$ & 8.17 *** & $-5.91 * * *$ & $18.62 * *$ \\
\hline $\mathrm{TC}_{\mathrm{t}-4}$ & $-114.5 * * *$ & $-3583.01 * * *$ & $9.9 * *$ & $241.57 *$ & $-3.33 * *$ & $3.26 * *$ & ns \\
\hline INF & 352.68 **** & ns & $-27.51 * *$ & ns & $26.61 * * *$ & $-17.64 * * *$ & $46.39 * *$ \\
\hline $\mathrm{INF}_{\mathrm{t}-1}$ & $385.0 * * *$ & ns & ns & ns & $-18.15 * *$ & $15.24 * *$ & $-67.11 * *$ \\
\hline $\mathrm{INF}_{\mathrm{t}-2}$ & Ns & $-13930 * * *$ & ns & $-787.69 * *$ & $\mathrm{~ns}$ & ns & $\mathrm{ns}$ \\
\hline $\mathrm{INF}_{\mathrm{t}-3}$ & $1009.74 * * *$ & ns & $50.54 * * *$ & ns & ns & $41 * * *$ & ns \\
\hline $\mathrm{INF}_{\mathrm{t}-4}$ & $-803.23 * * *$ & $-12841.8 * * *$ & $-37.98 * * *$ & ns & $-14.8 * *$ & $-26.61 * * *$ & $25.12 *$ \\
\hline EXP & 93.96 *** & $1354.98 * * *$ & $3.55 * * *$ & $-106.16 * *$ & ns & $0.86 * *$ & $-2.5 * * *$ \\
\hline $\mathrm{EXP}_{\mathrm{t}-1}$ & $-94.94 * * *$ & ns & ns & ns & $-1.12 * * *$ & ns & ns \\
\hline $\mathrm{EXP}_{\mathrm{t}-2}$ & $-91.30 * * *$ & $363.41 * *$ & $0.56^{* * *}$ & ns & $-2.47 * *$ & ns & $2.2 * * *$ \\
\hline $\mathrm{EXP}_{\mathrm{t}-3}$ & $\mathrm{Ns}$ & $247.47 * * *$ & ns & $-69.87 * *$ & $-2.01 * * *$ & $2.03 * * *$ & $-3.25 *$ \\
\hline $\mathrm{EXP}_{\mathrm{t}-4}$ & $28.13 * * *$ & $525.71 * *$ & $-3.73 * * *$ & $-79.14 *$ & $1.73 * * *$ & $-3.21 * * *$ & $\mathrm{~ns}$ \\
\hline PETR & $-17.74 * * *$ & $102.98 * *$ & $-1.48 * * *$ & $\mathrm{~ns}$ & $-0.87 * * *$ & ns & $-1.50 *$ \\
\hline PETR $_{t-1}$ & Ns & $254.76 * * *$ & $-0.57 * *$ & $-16.83 *$ & ns & $\mathrm{ns}$ & $-0.96 *$ \\
\hline PETR $_{\mathrm{t}-2}$ & $8.85 * * *$ & ns & ns & ns & $0.489 * *$ & $1.21 * * *$ & ns \\
\hline PETR $_{t-3}$ & Ns & $\mathrm{ns}$ & ns & $18.76 * *$ & ns & ns & $\mathrm{ns}$ \\
\hline PETR $_{t-4}$ & $-13.31 * * *$ & ns & ns & ns & $\mathrm{ns}$ & ns & ns \\
\hline IMP & Ns & $-3117.15 * * *$ & ns & ns & ns & $-2.3 *$ & $11.34 * *$ \\
\hline $\mathrm{IMP}_{\mathrm{t}-1}$ & $132.7 * * *$ & $-3880.35 * * *$ & $-8.88 * * *$ & $-138.55 *$ & $2.55 * *$ & ns & ns \\
\hline $\mathrm{IMP}_{\mathrm{t}-2}$ & $-96.4 * * *$ & $1490.56 * * *$ & ns & $-202.83 * *$ & ns & ns & ns \\
\hline $\mathrm{IMP}_{\mathrm{t}-3}$ & $-128.1 * * *$ & $1728.09 * * *$ & $-6.67 * * *$ & $-256.8 * *$ & $-3.48 * *$ & $-3.36^{* * * *}$ & $-9.68 *$ \\
\hline $\mathrm{IMP}_{\mathrm{t}-4}$ & $\mathrm{Ns}$ & $3815.08 * * *$ & $-4.76 * *$ & ns & ns & ns & $3.51 * *$ \\
\hline $\mathrm{R}^{2}$ ajustada & 0.87 & 0.90 & 0.84 & 0.31 & 0.63 & 0.93 & 0.51 \\
\hline Durbin-Watson & 1.87 & 2.54 & 2.61 & 1.88 & 1.93 & 2.01 & 1.49 \\
\hline C. de Schwarz & 599.67 & 766.70 & 330.33 & 701.26 & 317.72 & 286.27 & 412.56 \\
\hline
\end{tabular}




\begin{abstract}
$\longrightarrow$ Notas: a) $* * *, * * *$, indican el 1\%, 5\% y 10\% de significatividad, respectivamente; $n$ s indica no significatividad. b) C. de Schwarz = criterio Bayesiano de Schwarz. c) La estimación se efectuó utilizando una matriz de errores robusta, ante la presencia de heteroscedasticidad y autocorrelación.
\end{abstract}

Como se puede apreciar en el cuadro 3, el crecimiento de las importaciones presenta el mayor impacto en la explicación de las primas de riesgo al arrojar coeficientes estadísticamente significativos en el 100\% de las emisoras bajo estudio. Tan sólo al considerar el tercer rezago de la variable, los resultados confirman la relación significativa con 17 emisoras, mientras que el valor contemporáneo y el primer rezago son significativos en la mitad de los casos. Por su parte, el segundo y cuarto rezagos muestran significancia estadística en el $42.86 \%$ y $35.71 \%$ de las emisoras de la muestra, respectivamente. En cuanto al nivel de significancia estadística de este factor de riesgo, tanto en su valor contemporáneo y/o sus valores rezagados, es importante señalar que en el $47.76 \%$ del total de coeficientes significativos se alcanza el $1 \%$ de significancia.

La segunda variable en importancia es la dinámica del tipo de cambio, al presentar significancia estadística sobre las primas de riesgo del $96.43 \%$ de las empresas de la muestra. Cabe destacar que del total de los coeficientes significativos asociados con el impacto cambiario sobre los spreads de la deuda de las empresas analizadas, el nivel de significatividad alcanza el máximo valor, es decir, un coeficiente de confianza del $99 \%$ en el $53.16 \%$ de los casos. El primero y cuarto rezagos impactan significativamente en el $64.29 \%$ y $60.71 \%$ de las primas de la muestra, respectivamente. El segundo rezago de la variable afecta al $57.14 \%$, mientras que tanto el valor contemporáneo de la variable, así como su tercer rezago muestran significancia en la mitad de las primas de las emisoras bajo estudio.

El tercer lugar, de acuerdo con su importancia en el impacto de las primas de riesgo, lo comparten las variables de crecimiento de la oferta monetaria medida por el agregado monetario M1, el PIB, la inflación y el crecimiento de las reservas internacionales al mostrar coeficientes significativos en el $92.86 \%$ de la muestra, es decir, en 26 de las 28 emisoras. Los resultados señalan que el nivel de significatividad de los parámetros calculados para estas cuatro variables alcanza el mayor nivel, es decir, el $1 \%$, en el $62.50 \%$ del total de coeficientes significativos en el caso del crecimiento de M1; el 56.94\% en el del crecimiento del PIB; $65.67 \%$ para la inflación y $48.33 \%$ en el caso del comportamiento de las reservas internacionales. 
El crecimiento del agregado monetario M1, tanto en su valor contemporáneo y/o sus valores rezagados, mostró impacto significativo en las primas de 26 empresas. Tanto el valor contemporáneo del comportamiento del M1 como su tercer rezago, contribuyen a explicar el crecimiento de las primas de riesgo (spreads) de 14 emisoras, es decir, 50\% de las incluidas en la muestra. Por otro lado, en el $43 \%$ de tales primas se encuentra una relación significativa hacia el primer y cuarto rezagos de la variable. El mayor impacto se aprecia en el segundo rezago al mostrar significancia en 21 emisoras, o sea, el $71.43 \%$ de los casos analizados.

Por otro lado, la dinámica del PIB impacta al $60.71 \%$ de las primas de riesgo de las emisoras estudiadas considerando el segundo rezago de la variable; le sigue el valor del cuarto rezago, afectando significativamente al $53.57 \%$ de los casos. Por su parte, el valor contemporáneo y el primer rezago muestran significancia en la mitad de las primas bajo estudio. El tercer rezago de la variable es el que presenta el menor impacto al resultar significativo en 12 emisoras. En cuanto al impacto de los cambios en la inflación, los resultados revelan que su valor contemporáneo afecta al $67.86 \%$ de las primas de las emisoras de la muestra mientras que el cuarto rezago al $64.29 \%$. Le siguen los valores de tercer y segundo rezagos con significancia en $42.86 \%$ y $35.71 \%$ de los casos, respectivamente. El menor impacto se asocia al primer rezago con impacto en $28.57 \%$ de las emisoras. Por otro lado, se observa que el mayor impacto del crecimiento de las reservas internacionales se percibe en el valor contemporáneo con impacto en $53.57 \%$ de las primas de riesgo, seguido del segundo y tercer rezagos que explican, cada uno, el comportamiento de las primas de la mitad de los casos. Por su parte, el cuarto rezago muestra significancia en $39.29 \%$ mientras que el primero en el $21.43 \%$ de las emisoras.

El cuarto lugar lo ocupa el crecimiento de las exportaciones, los resultados confirman una relación significativa en $89.29 \%$ de las primas de las emisoras bajo estudio, es decir, en 25 de las 28 empresas. Los principales impactos se perciben en los valores contemporáneo, así como en el tercer y cuarto rezagos, al mostrar significancia en $60.71 \%, 57.14 \%$ y $50 \%$ de los casos, respectivamente. El menor impacto de las primas de riesgo se aprecia en el segundo y primer rezagos, con significancia estadística en el $42.86 \%$ y $28.57 \%$ de los casos, respectivamente. En cuanto al nivel de significancia estadística del efecto del crecimiento de las exportaciones sobre las primas de riesgo, tanto en su valor contemporáneo y/o sus valores rezagados, es importante señalar que en el $49.25 \%$ de los parámetros significativos estimados el nivel de confianza es del $99 \%$. 
La variable relativa a la tasa de cambio en los precios del petróleo ocupa el último lugar en cuanto al número de emisoras que impacta significativamente. Los resultados muestran que la dinámica de los precios petroleros afecta a las primas de riesgo de 22 emisoras, es decir, al 78.57\%. Los parámetros estimados alcanzaron el $1 \%$ de significatividad en el $54.35 \%$ de los casos analizados.

Por otro lado, los resultados muestran que las primas de riesgo de las siguientes 17 emisoras se ven afectadas por todos los factores de riesgo modelados: VITRO, TELMEX, TELEVISA, SAB, REALTUR, LIVERPOL, GIGANTE, GIISA, GMARTI, GMODERN, COMERCI, ECKO, GCARSO, BIMBO, ALFA, ARISTOS, AUTLAN.

Asimismo, las emisoras que responden a siete de los ocho factores de riesgo propuestos son: TMM, SANLUIS, PEÑOLES, ICA, GRUMA, GCC, CEMEX, ACCEL; siendo la variable excluida en el modelo restringido: PIB, MI, PETR, EXP, PIB, PETR, PETR E INF, respectivamente. Por su parte, las primas de riesgo de AHMSA y POSADA mostraron una relación significativa con el $62.50 \%$ de los factores de riesgo propuestos, quedando excluidas TC, EXP y PETR, en el caso de la primera emisora y M1, RESINT y PETR en la segunda. Finalmente, las primas de BEVIDES se explicaron con un menor número de factores de riesgo, al mostrar significancia estadística respecto al 50\% de ellos y quedando fuera del modelo: INF, EXP, RESINT y PETR.

$\mathrm{Al}$ analizar la relación entre los factores de riesgo y las primas de las emisoras de la muestra, agrupadas por sectores de actividad económica, se encuentra que las primas de riesgo de las emisoras analizadas del sector comercial se ven influidas, principalmente, por el comportamiento del agregado monetario y la dinámica del tipo de cambio presentando en ambos casos, coeficientes significativos en el $66.67 \%$ de los casos analizados. En cuanto a las emisoras pertenecientes al sector de comunicaciones y transportes, los resultados revelan que las variables más importantes son el comportamiento del tipo de cambio, del M1 y de los precios con impactos significativos en el $73.33 \%, 66.67 \%$ y $53.33 \%$ de los casos, respectivamente. Por su parte, las primas de riesgo del sector de la construcción responden principalmente al tipo de cambio y las importaciones, con impactos significativos en el $66.67 \%$ y $53.33 \%$ de los casos, respectivamente; mientras que las primas del sector de transformación al crecimiento de las reservas internacionales y de las exportaciones con $63.33 \%$ y $53.33 \%$, respectivamente. Las primas de riesgo de las emisoras de la industria extractiva son sensibles a la inflación en el $80 \%$ de los casos y, con igual importancia, al crecimiento de la economía, la dinámica cambiaria y de las exportaciones con un $60 \%$ de coeficientes significativos. En el caso 
del sector de varios, las primas de riesgo responden principalmente al crecimiento económico y el de las importaciones con $64 \%$ y $56 \%$ de los casos con impactos significativos, respectivamente. Por último, las tasas de crecimiento del M1, del PIB, de las exportaciones y de la inflación, presentan la misma importancia sobre las primas de riesgo del sector de servicios al presentar el 53.33\% de los coeficientes significativos.

Finalmente, en promedio la mayor $\mathrm{r}^{2}$ ajustada se observa en los sectores de la industria extractiva y de comunicaciones y transportes, con valores de .64 y .62, respectivamente, seguidos de los sectores de varios con .59 y servicios y transformación, ambos con un .54. Por otro lado, se encuentran el sector comercial y de la construcción con valores $\mathrm{r}^{2}$ de .50 y .30 , respectivamente. Cabe destacar que el valor del coeficiente de determinación ajustado supera el .57 en el $43 \%$ de los casos bajo estudio, llegando incluso a valores de .9 y .93 en los casos de SAB y TMM, respectivamente.

\section{Conclusiones}

El análisis realizado presenta evidencia de la relación entre variables macroeconómicas, como representantes del riesgo sistemático y el comportamiento de las primas de riesgo del costo de la deuda de los principales corporativos de la economía mexicana. Es importante señalar que la evidencia de estudios que abordan la relación entre dichas primas de riesgo, calculadas a partir de la información financiero-contable de la empresa y el riesgo sistemático es escasa; hasta donde sabemos éste es el primer intento de abordar el tema en el mercado mexicano. El estudio contribuye a la literatura financiera al menos en tres aspectos.

En primer lugar, se presenta una forma novedosa de calcular la prima de riesgo o spread del crédito, lo que supone ventajas para las empresas mexicanas que al no ser calificadas por agencias especializadas carecen de elementos para conocer su nivel de riesgo, pero que mediante los resultados presentados pueden mejorar su entendimiento de la forma en que el riesgo sistemático doméstico afecta el comportamiento de las primas de riesgo, construidas estas últimas a partir de la información financiero-contable y no con base en datos de mercado. Por otro lado, los resultados presentados pueden servir a reguladores e inversionistas; al abrir posibilidades de análisis sin implicar necesariamente, altos costos de transacción y/o económicos. 
En segundo lugar, este estudio documenta la relación significativa entre las primas de riesgo del costo de la deuda, de emisoras del mercado mexicano y los factores de riesgo propuestos, como representantes domésticos del riesgo sistemático. El análisis revela que la totalidad de dichos factores, ya sea en sus valores contemporáneos o rezagados ${ }^{15}$, son importantes en la modelación de las primas de riesgo en México. Cabe destacar que el factor de riesgo que parece afectar a una menor proporción de primas de riesgo de las empresas estudiadas, la evolución de los precios del petróleo, presentó significancia estadística en casi el 80\% de los casos al afectar el spread de 22 emisoras. Los demás factores de riesgo contribuyeron a explicar los spreads de 25 empresas o más, llegando incluso a explicar el total de las primas de la muestra. En este sentido, el análisis que se ha presentado en estas páginas contribuye a proporcionar evidencia empírica adicional a la que han ofrecido otros investigadores sobre las características del riesgo sistemático en México.

Como tercera contribución, este estudio ofrece evidencia empírica a favor de los argumentos de la relevancia de las importaciones como fuente de riesgo sistemático aparte de las exportaciones, como lo propone López (2006a y 2006b). Como se mostró en el análisis empírico presentado, los spreads de todas las emisoras de la muestra analizada muestran sensibilidad estadísticamente significativa al comportamiento de dicha variable.

Naturalmente, debido a la importancia que el crédito tiene para las empresas y la economía en general como factor impulsor de la actividad productiva, se requiere de más estudios que aborden el vínculo del riesgo de crédito y el riesgo sistemático. No obstante, el alto nivel de significancia estadística presentada en este estudio, la literatura señala la posibilidad de enriquecer la modelación incluyendo otros factores como la situación financiera de la empresa, pero por el momento diferimos esa tarea para un trabajo futuro.

\footnotetext{
${ }^{15} \mathrm{Si}$ bien es cierto que la formulación de sistemas de alertas tempranas está más allá del objetivo de este estudio, los resultados muestran que los valores rezagados de los factores de riesgo sistemático analizados en este documento podrían servir como base para instrumentar tales modelos de alerta temprana.
} 


\section{Referencias}

Al-Shanfari, Hatem (2003). "Testing the Arbitrage Pricing Theory in net oil exporting countries". Ponencia. European Applied business Research Conference. Venice.

Altman, Edward I. y Anthony Saunders (1998). "Credit Risk Measurement: Developments over the last 20 years". Journal of Banking \& Finance, Vol. 21. pp. 1721-1742.

(2005). "An emerging market credit scoring system of corporate bonds”. Emerging Markets Review, Vol. 6, pp. 311-323.

y Mario Onorato (2004). "An Integrated Pricing Model for Defautable Loans and Bonds". European Journal of Operations Research, pp. 65-82.

Benell, Julia A., David Crabbe, Stephen Thomas y Owain Gwilym (2006). "Modelling sovereign credit ratings: Neural networks versus ordered probit". Expert Systems with Aplications, Vol. 30, pp. 415-425.

Bielecki, Tomasz R. y Marek Rutkowski (2000). "Multiple Ratings Model of Defautable Term Structure". Mathematical Finance, Vol. 10, No. 2, pp. 125-139.

Bank of International Settlements (BIs) (1999). "Credit Risk Modelling: Current Practice and Applications". Basel Committee on Banking Supervision.

Cagnetti, Arduino (2002). "Capital Asset Pricing model and Arbitrage Pricing Theory in the Italian Stock Market: an empirical evidence". Mimeo.

CANTOR, RichaRd (2004). "An introduction to recent research on credit ratings". Journal of Banking and Finance, Vol. 28, pp. 2565-2573.

Carey, Mark y Mark Hrycay (2001). "Parameterizing credit risk models with rating data". Journal of Banking \& Finance, Vol. 25, pp. 197-270. 
Chan, Konan y Narasimhan Jegadeesh (2004). "Market-Based Evaluation for Models to Predict Bond Ratings". Review of Pacific Basin Financial Markets and Policies, Vol. 7, pp. 153-172.

Crouhy, Michel, Dan Galai y Robert Mark (2000). "A Comparative Analysis of Current Credit Risk Models". Journal of Business \& Finance, Vol. 24, pp. 59-117.

(2001). "Prototype risk rating system". Journal of Banking \& Finance, Vol. 25.

De la Calle, Luis F. (1991). "Diversification of Macroeconomic Risk and International Integration of Capital Markets: The Case of Mexico". The World Bank Economic Review.

Delianedis, Gordon y Robert Geske (2001). "The Components of Corporate Credit Spreads: default, recovery, tax, jumps, liquidity and market factors". Anderson Graduate School of Management. University of California, Los Ángeles. Working Paper 22-01.

(2003). "Credit Risk and Neural Default Probabilities: Information about Rating Migrations and Defaults". European Finance Association; Annual Conference, Paper No. 962.

Doshi, Kokila, Robert Johnson, Edgar Ortiz y Luc Soenen (2001). "Privatization, liberalization and stock market performance: the case of Mexico". En Kotabe, Masaaki y Leal, Ricardo P. C. (edit.). "Market revolution in Latin American: Beyond Mexico". Pergamon. Kidlington, Oxford.

Eiteman, David K., Arthur I. Stonehill y Michael H. Moffett (2001). “Multinational Business Finance”. 9a. ed., Addison Wesley Longman, Pearson Education; 9th edition.

Farnsworth, Heber y Tao Li (2007). "The Dynamics of Credit Spreads and Ratings". Journal of Financial and Quantitative Analysis, Vol. 2, No. 3, pp. 595-620. 
Frenkel, Roberto (2003). "Globalización y crisis financieras en América Latina”. Revista de Economía Política, Vol. 23, No 3, pp. 94-111.

Gentry, James A., Michael J. Shaw, Antoinette C. Tessmer y David T. WhiTFORD (2002). "Using Inductive Learning to Predict Bankruptcy". Journal of Organizational Computing and Electronic Commerce, Vol. 12, pp. 39 - 57.

Gilbert, Lisa R., Krishnagopal Menon y Kenneth B. Schartz (1990). "Predicting Bankruptcy for Firms in Financial Distress". Journal of Business Finance \& Accounting, Vol. 17, pp. $161-171$.

Gupton, G.M., C.C. Finger y M. Bhatia (1997). "CreditMetrics-Technical Document". J.P. Morgan \& Co. Incorporated, New York.

Hanson, Sammuel y Til Schuermann (2006). "Confidence Intervals for Probabilities of Default". Journal of Banking \& Finance, Vol. 30, pp. 22812301.

Hull, John, Mirela Predescu y Alan White (2004). “The relationship between credit default swap spreads, bond yields, and credit rating announcements". Journal of Banking \& Finance, Vol. 28, pp. 2789-2811.

Johnston, Jack y John DiNardo. (1997). “Econometric Methods", $4^{\text {th }}$ edition, Mc. Graw-Hill International Editions, Singapore.

Krahnen, Jan Pieter y Martin Weber (2001). "Generally Accepted Rating Principles: A Primer”. Journal of Banking and Finance, Vol. 25, pp. 3-23.

Livingston, Miles, Andy Naranjo y Lei Zhou (2005). "Information Asymmetry, Bond Split Rating, and Rating Migration”. Financial Management Association; International Annual Meeting, Chicago Ill.

LÖFFLER, GUNTER (2004). "An anatomy of rating through the cycle". Journal of Banking \& Finance, Vol. 28, pp. 695-720.

López Herrera, Francisco (2006a). "Riesgo sistemático en el mercado mexicano de capitales: un caso de segmentación parcial". Contaduría y Administración, No. 219, pp. 85-113. 
(2006b). "Factores macroeconómicos y riesgo sistemático: modelos multifactoriales de los mercados de capitales del TLCAN". Tesis, Doctorado en Economía, Facultad de Economía, Universidad Nacional Autónoma de México.

y Francisco J. VÁzquez Téllez (2002). "Variables económicas y un modelo multifactorial para la Bolsa Mexicana de Valores: análisis empírico sobre una muestra de activos". Revista Latinoamericana de Administración, No. 29, CLAdEA, Bogotá, pp. 5-28.

Moncarz, Elisa S., Raúl Moncarz, Alejandra Cabello y Benjamín MonCARZ (2006). "The Rise and Collapse of Enron: Financial Innovation, Errors and Lessons". Contaduría y Administración, No. 218, pp. 17-37.

Nava Peralta, Noel (1996). "The Arbitrage pricing Theory: An application for the Mexican Stock Exchange". Mimeo. Instituto Tecnológico y de Estudios Superiores de Monterrey, Campus Ciudad de México.

Navarro, Cora y Roberto Santillán Salgado (2001). "A test $\mathrm{f}$ the APT in the mexican stock market”. BALAS Conference. Universidad de San Diego, California.

Otero, José María (1993). “Econometría. Series temporales y predicción”. Editorial AC. Madrid.

Partnoy, Frank (2001). "The Paradox of Credit Rating”. University of San Diego. Law and Economics Research Papers Series, Working Paper No. 20.

Prysock, Mark (2006). "Why FEI Supports Credit Rating Agency Reform”. Financial Executive, Vol. 22, Issue 4, p. 63.

Segoviano Basurto, Miguel A. y Pablo Padilla (2006). "Portfolio Credit Risk and Macroeconomic Shocks: Applications to Stress Testing Under DataRestricted Environments". International Monetary Fund; Working Paper 06/283, December.

Selamat, Zarehan (2001). "Empirical test of macroeconomic variables and stock market returns in Asian emerging markets". Faculty of Management, Multimedia University. Mimeo. 
Sy, Amadou N. R. (2002). "Emerging market bond spreads and sovereign credit ratings: reconciling market view with economic fundamentals". Emerging Markets Review, Vol.3, pp. 380-408.

(2003). "Rating the Rating Agencies: Anticipating Currency Crises or Debt Crises". IMF, International Capital Markets Departaments, Working Paper Series; WP/03/122, pp. 2845-2867.

Van den Goorbergh, Rob W. J., Frans A. De Roon y Bass J. Werker (2003). "Economic hedging portfolio". Working Paper. Finance Department and Center for Economic Research, Tilburg University.

WeI, JAson Z. (2003). “A Multi-Factor, Credit Migration Model for Sovereign and Corporate Debts". Journal of International Money and Finance, Vol. 22, pp. 709-735.

Wendin, Jonathan y Alexander McNeil (2006). "Dependent Credit Migrations". National Centre of Competente in Research; Financial Valuation and Risk Management Working Paper Series, No. 182. 